Elsevier Editorial System(tm) for Planetary and Space Science Manuscript Draft

Manuscript Number:

Title: THE C1XS X-RAY SPECTROMETER ON CHANDRAYAAN-1.

Article Type: Research Paper

Keywords: Moon

Lunar Composition

$X$ ray spectroscopy

Chandrayaan-1

Space Instrumentation

Corresponding Author: Prof. Manuel Grande,

Corresponding Author's Institution: Aberystwyth University

First Author: Manuel Grande

Order of Authors: Manuel Grande; Brian Maddison; chris J Howe; Barry J Kellett; P Shreekumar; Juhani Huovelin; Ian Crawford; Lionel C d'Uston; David Smith; Manesh Anand; Narendra Bhandari; Antony T Cook; Vera Fernandes; Bernard Foing; Olivier Gasnault; J N Goswami; Andrew Hollang; Katie H Joy; Detlev Kochney; David Lawrence; Sylvestre Maurice; T Okada; Syama Narendrath; Carle Pieters; David Rothery; Sara S Russell; Alok Shrivastava; Bruce Swinyard; Martin Wilding; Mark Wieczorek

Abstract: The Chandrayaan-1 X-ray Spectrometer (C1XS) is a compact X-ray spectrometer for the Indian Space Research Organisation (ISRO) Chandrayaan-1 lunar mission. It exploits heritage from the D-CIXS instrument on ESA's SMART-1 mission. As a result of detailed developments to all aspects of the design, its performance as measured in the laboratory greatly surpasses that of D-CIXS. In comparison with SMART-1, Chandrayaan-1 is a science oriented rather than a technology mission, leading to far more favourable conditions for science measurements. C1XS is 
designed to measure absolute and relative abundances of major rock-forming elements (principally $\mathrm{Mg}, \mathrm{Al}, \mathrm{Si}, \mathrm{Ca}$ and $\mathrm{Fe}$ ) in the lunar crust with spatial resolution $\leq 25 \mathrm{FWHM} \mathrm{km}$, and to achieve relative elemental abundances of better than $10 \%$. 


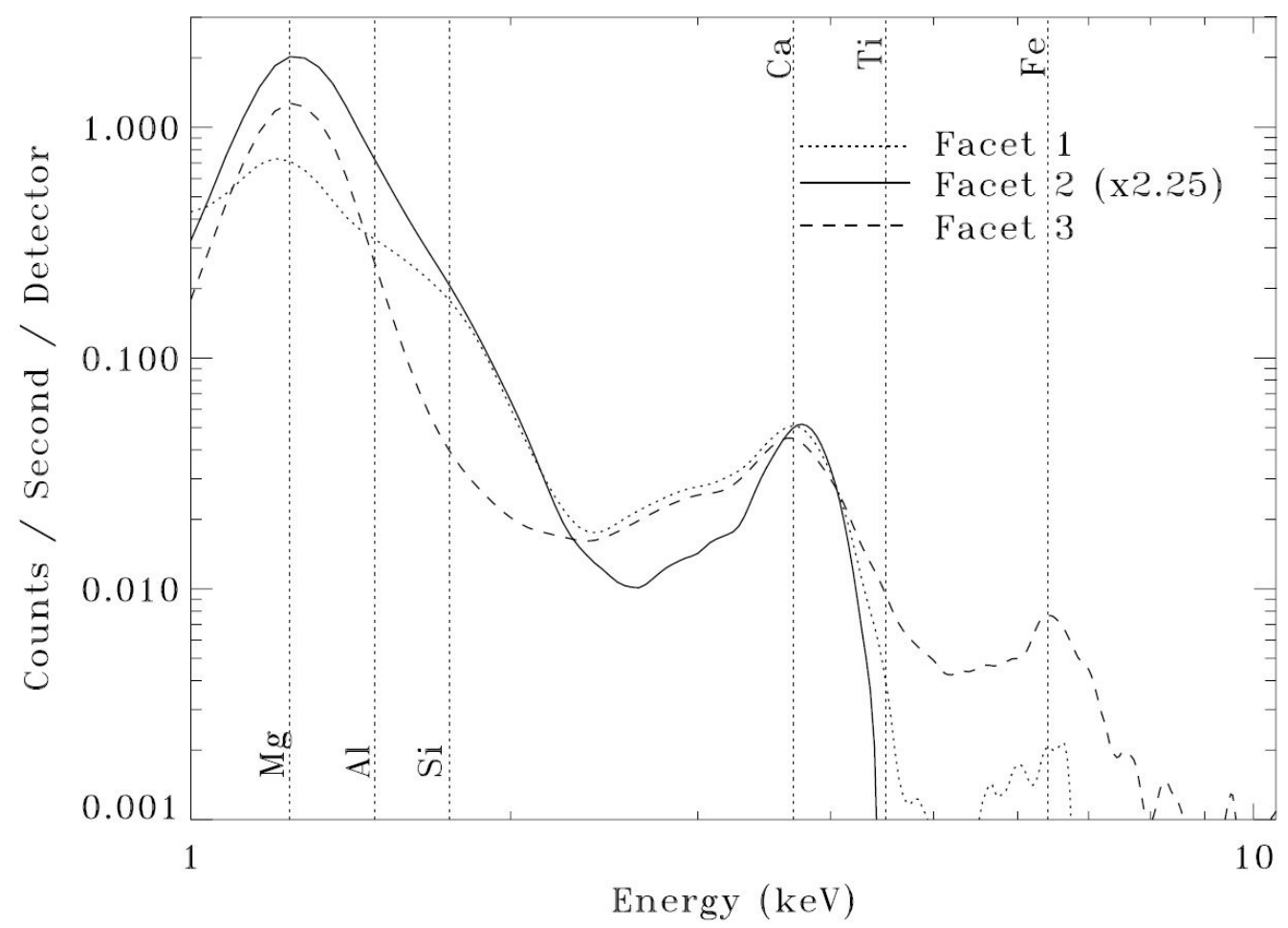

a)

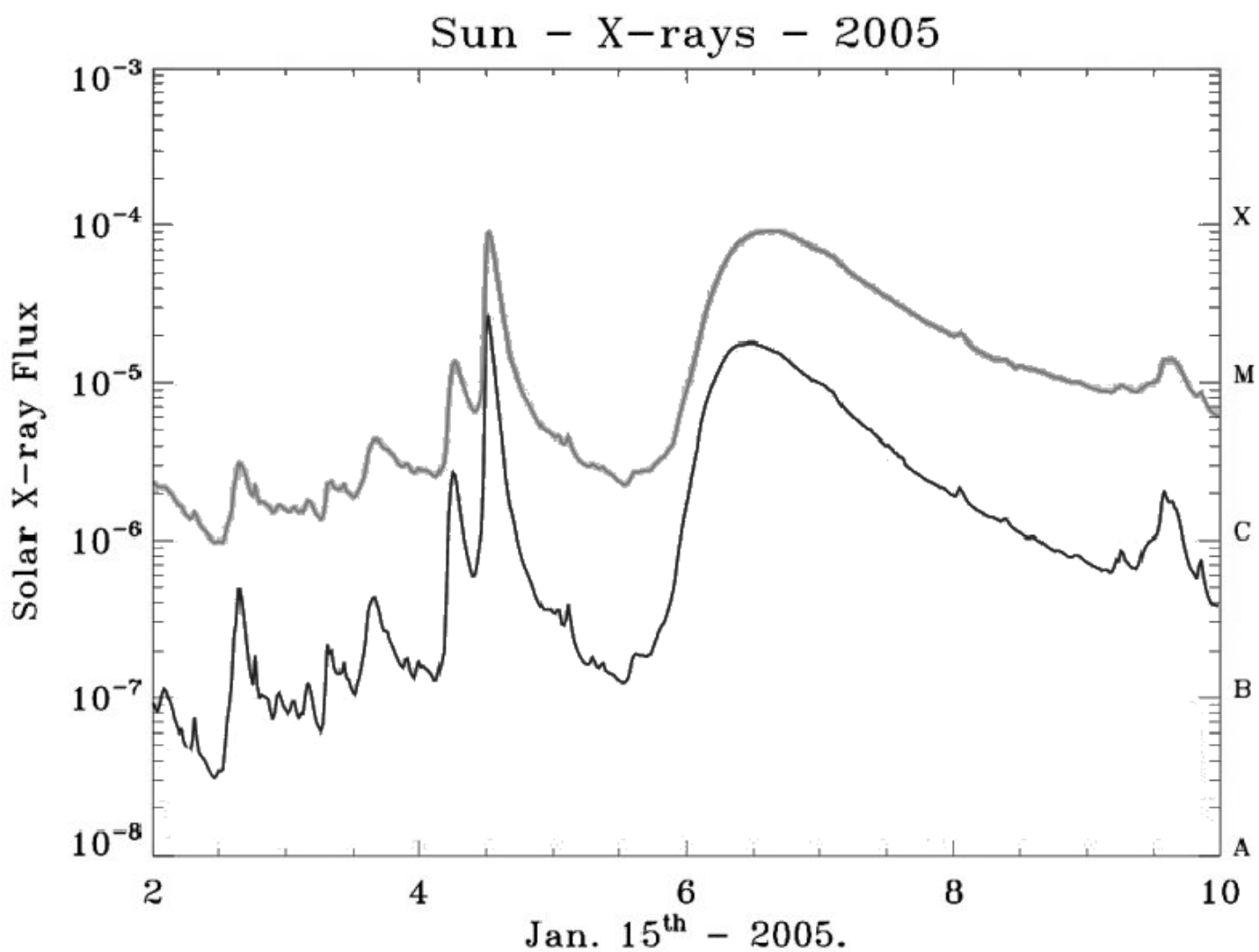

b)

Figure 1: a) Fluorescence spectra obtained by D-CIXS on SMART-1 on 15 Jan 2005, indicating an ability to remote sense elements in the top few micrometers of the Lunar regolith, in particular Mg, Al, Si, Ca and Fe, as indicated by vertical lines (Grande et al 2007). Vertical lines indicate the expected position of these elements. b) Also shown is the $10 \AA$ (black) and $1 \AA$ (grey) (TBC) Solar X-ray illumination at the time, derived from GOES data, indicating the high variability of the Sun as an X-ray source. Times are indicated on the X axis in hours. Note the conventional A,B,C,M,X nomenclature for flare levels are indicated on the left hand side of the plot. 


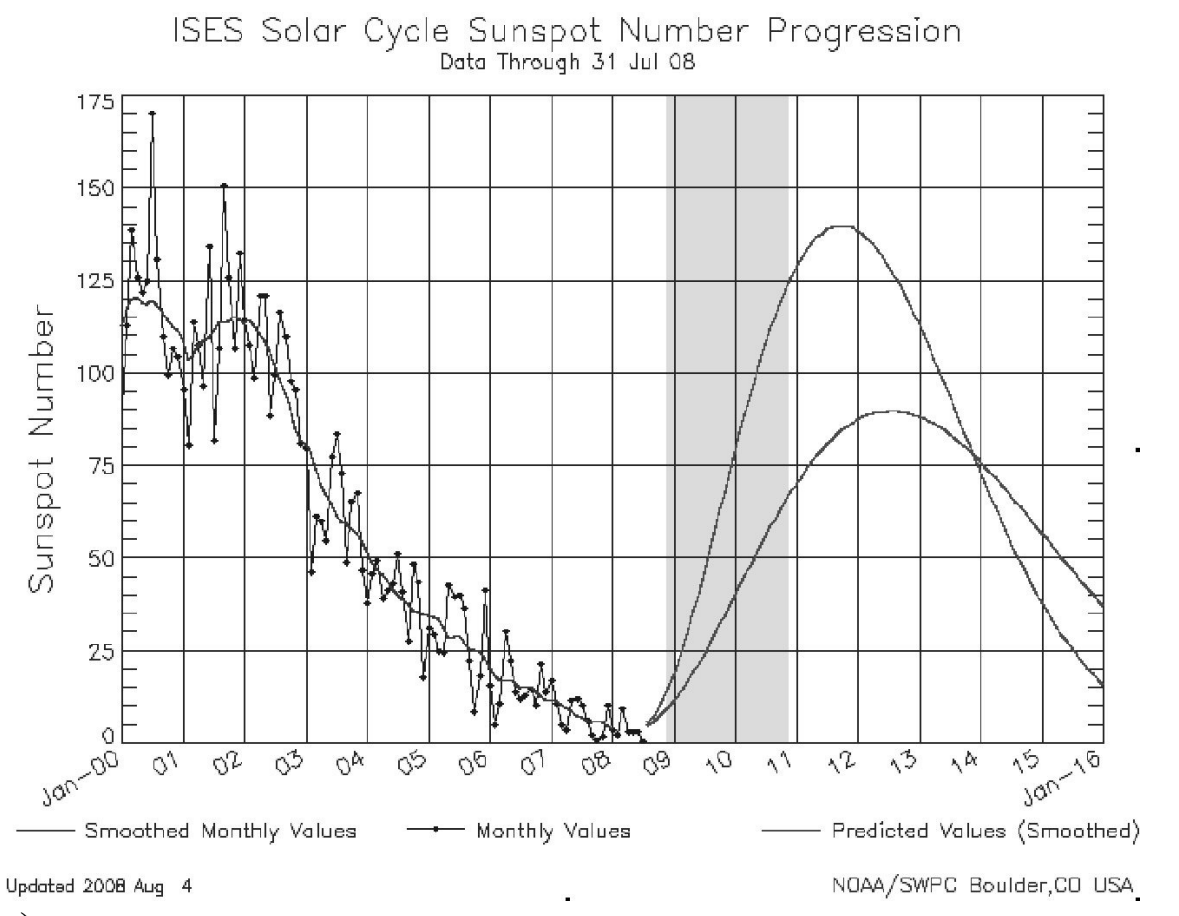

a)

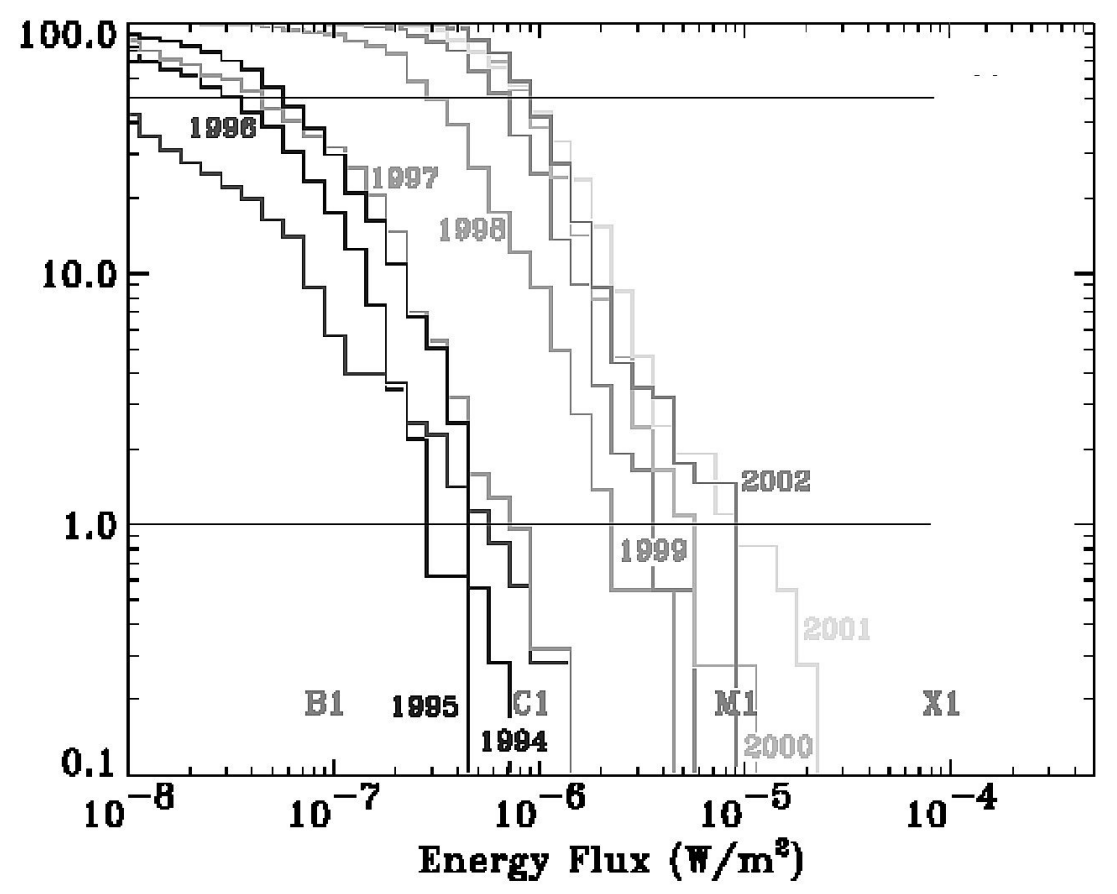

b)

Figure 2 (a) Past blue and predicted red solar cycle variation (NOAASpace Weather Prediction Center) during the Chandrayaan-1 missions (Ref). Note that whilst SMART-1 took place during a decline into solar minimum, Chandrayaan-1 will be launched in the ascending phase of the cycle (shown by shaded region), which is predicted to be close to peak by the end of the mission. The two red lines indicate alternative predictions.

(b) cumulative distribution of one minute solar flare date from the previous cycle, indicating probability of illumination above a certain illumination level more. To obtain predicted fluxes add 11 to the year. 

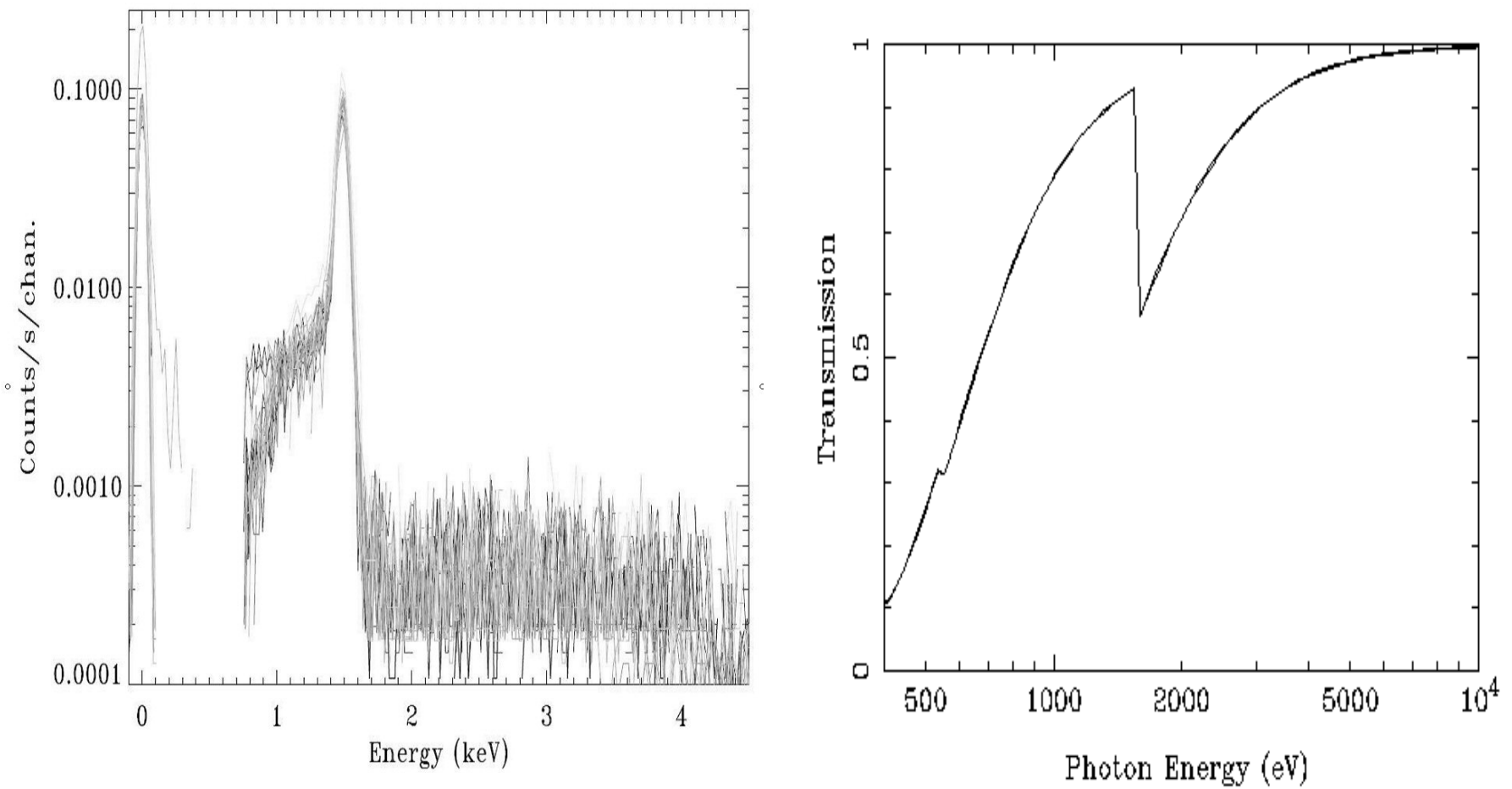

a)

b)

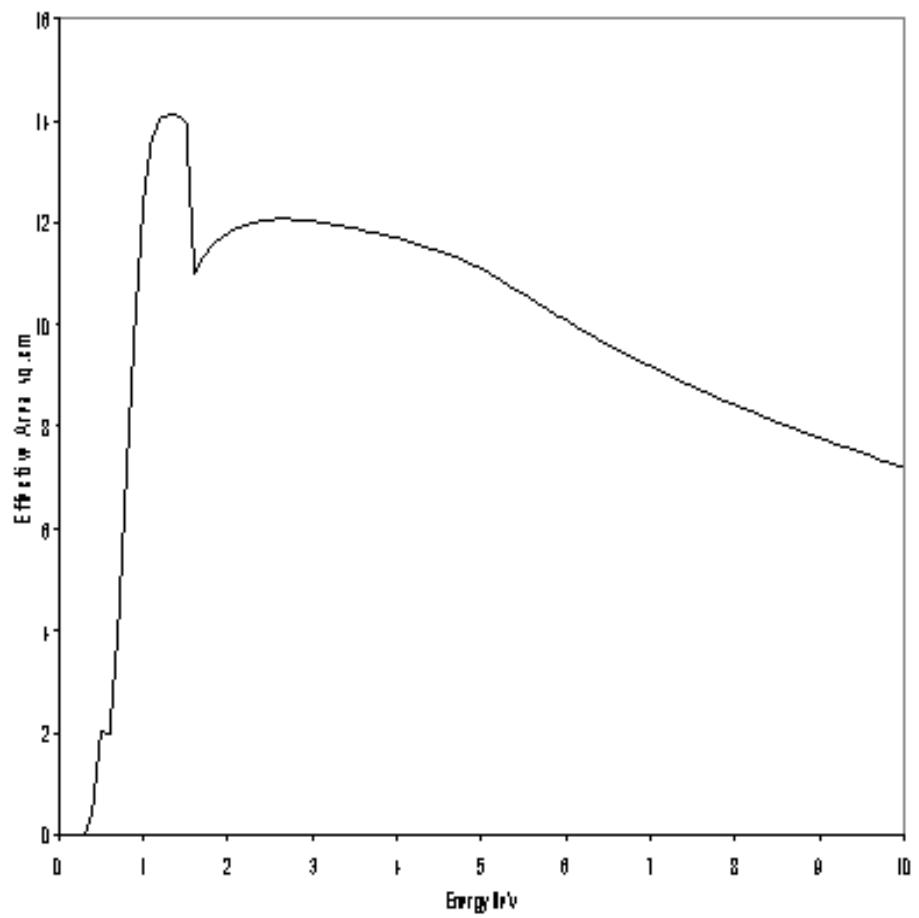

c)

Figure 3: (a)Measured performanceat low energies in C1XS illustrating the reponse of all 24 SCDs to $\mathrm{Al} \mathrm{K \alpha}(1.487 \mathrm{keV})$ and the low energy cut off of the instrument at $750 \mathrm{eV}$, derived during calibration (Kellett et al forthcoming). Note the excellent alignment and uniformity of the 24 different responses. (b) calculated filter transmission for $800 \mathrm{~nm}$ of $\mathrm{Al}$ coated Polyimide over the active range of the instrument. (c) Calculated effective area of the instrument, plotted against energy, based on geometry, and filter and detector specification, but neglecting electronic losses. Note the rapid loss of effective area below $1 \mathrm{keV}$. 


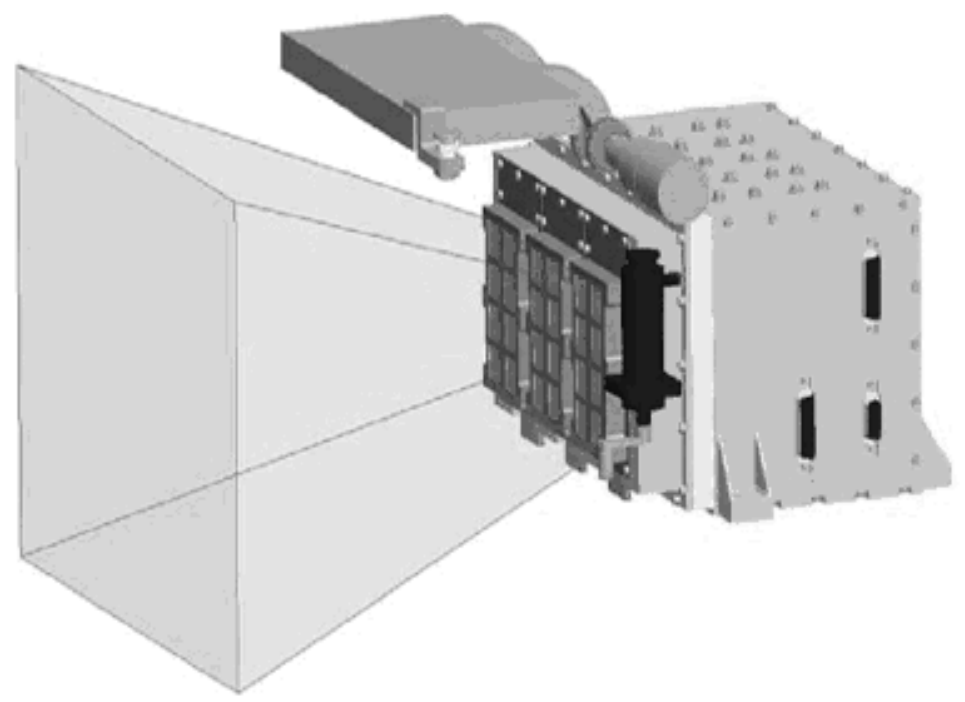

Figure 4: CAD image of the C1XS instrument showing coalligned front detectors, deployable radiation shield and $14^{\mathrm{O}}$ Field of View. Note light coloured thermal gasket separating cool detector enclosure from electronics case to the right.

The instrument design aims to keep detector temperatures below $-17.5 \mathrm{C}$, which ensures optimum signal to noise and stability, as well as improving radiation tolerance. 
Figure 5

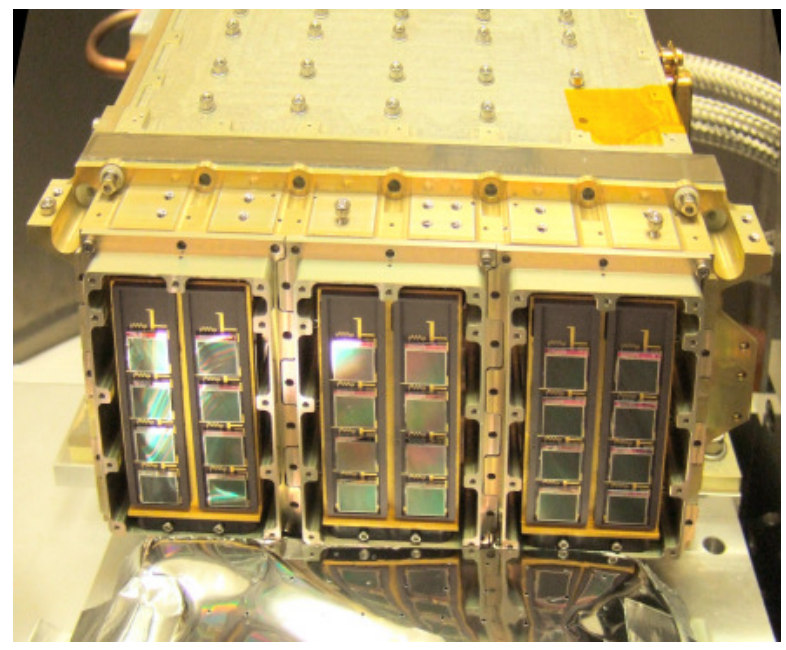

Figure 5: View of the C1XS flight instrument during calibration. The collimator assembly and doors have not yet been added, so that the 24 swept charge detectors, arranged in ladders of four, are clearly seen. 


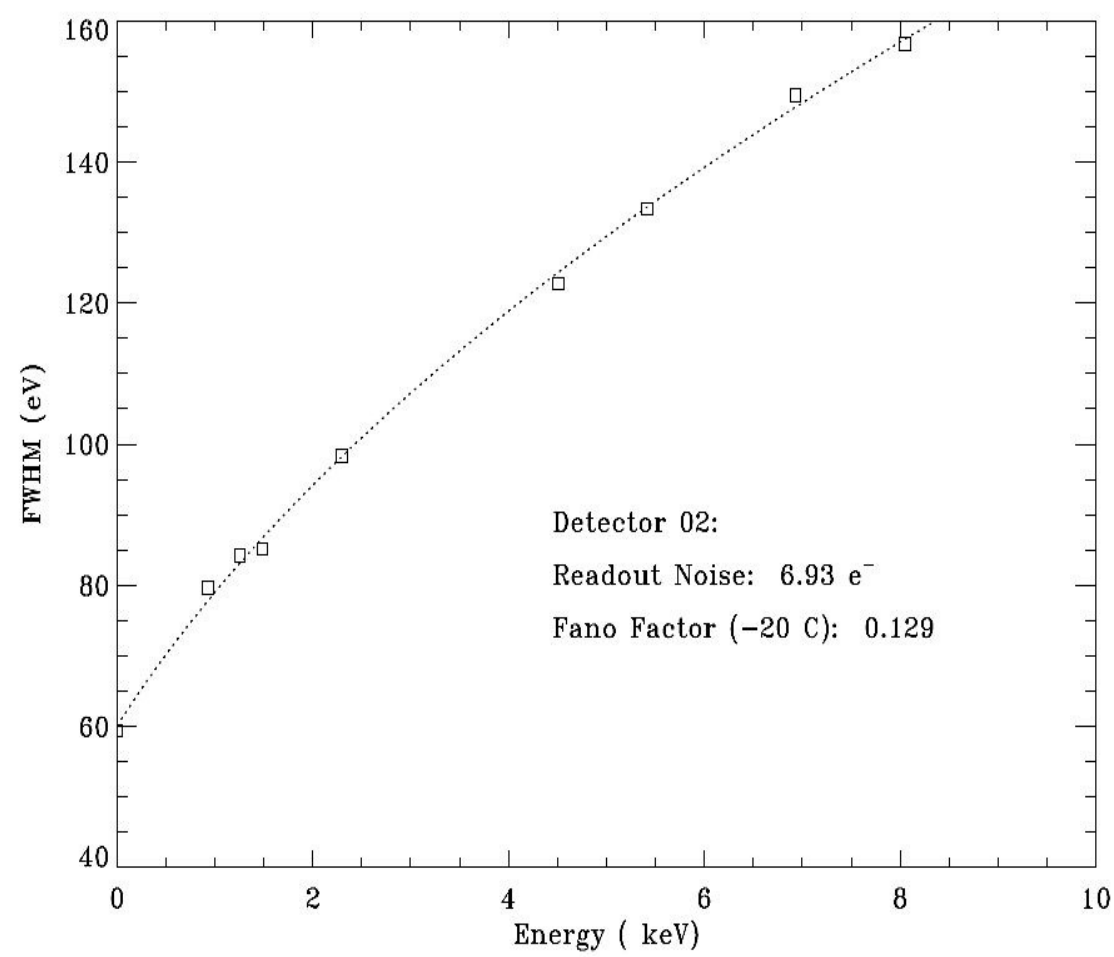

a)

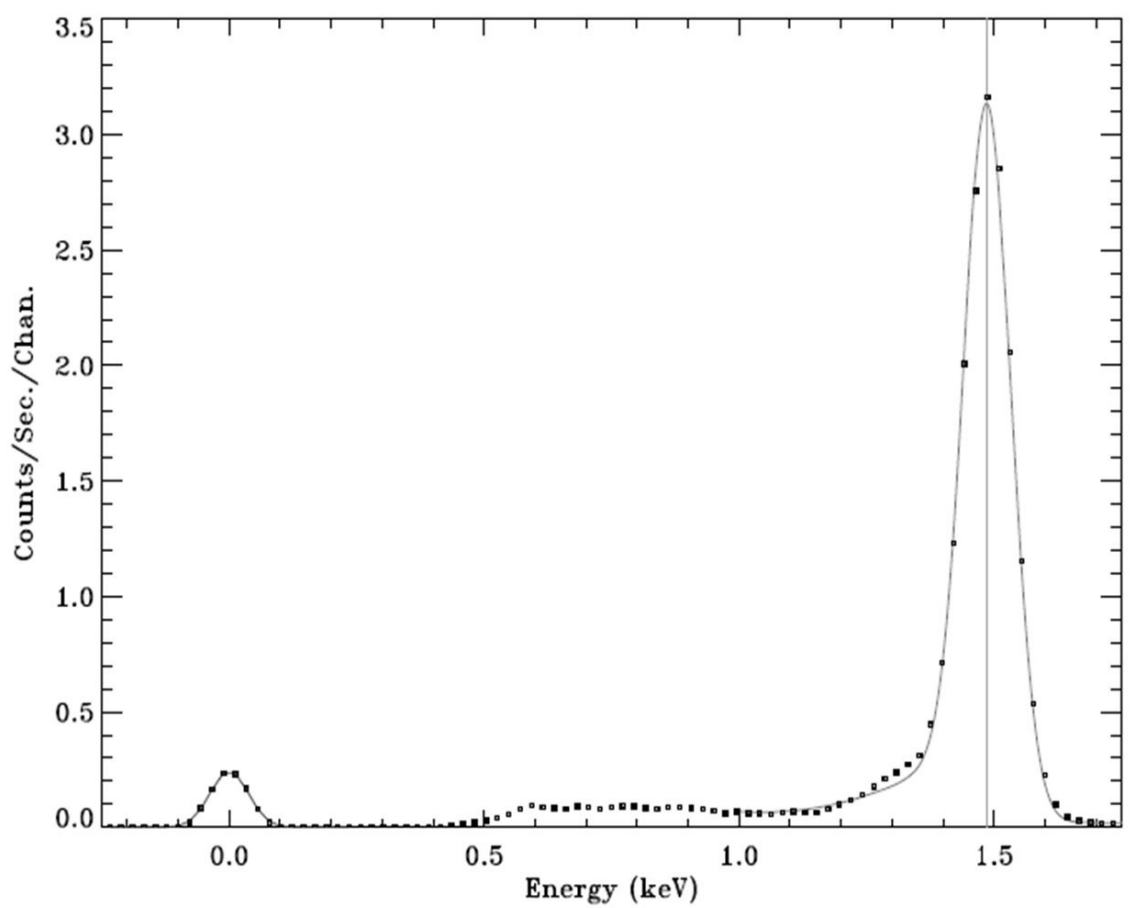

b)

Figure 6: Laboratory performance of C1XS as obtained during calibration (Kellett et al 2008). (a) Measured combined FWHM of the detectors and readout electronics as a function of energy. (b) Example showing measured resolution at the $1.49 \mathrm{keV}$ Aluminium $\mathbf{K} \alpha_{\mathbf{1}}$ line and also the well separated zero energy electronic noise peak. Note the major improvement over the performance of D-CIXS shown in Figure 1. 


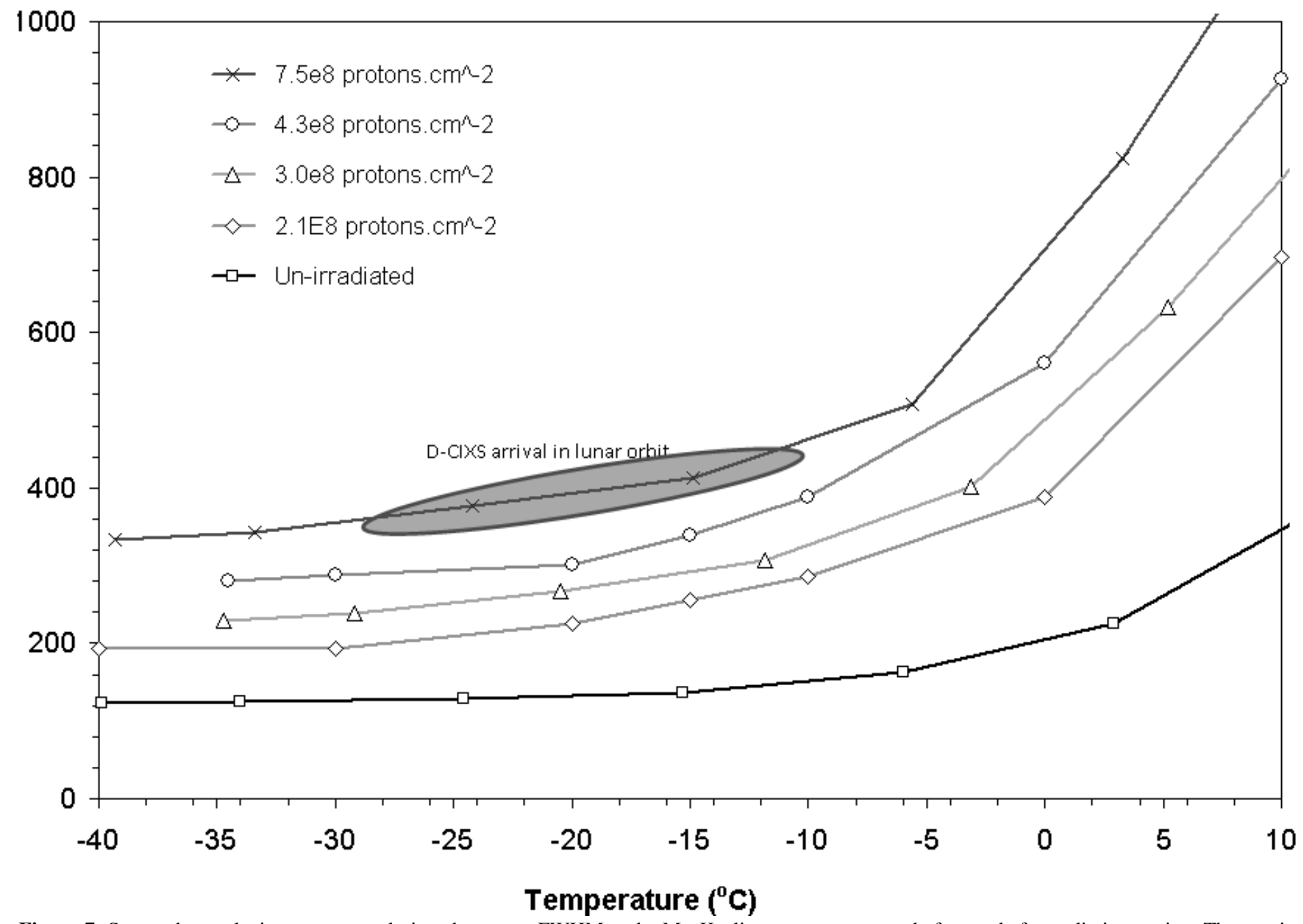

Figure 7: Swept charge device energy resolution shaown as FWHM at the Mn-K $\alpha$ line vs. temperature, before and after radiation testing. The specified maximum operating temperature is $-17.5^{\circ} \mathrm{C}$ Note the favourable comparison with D-CIXS FWHM shown in between the dashed lines. 


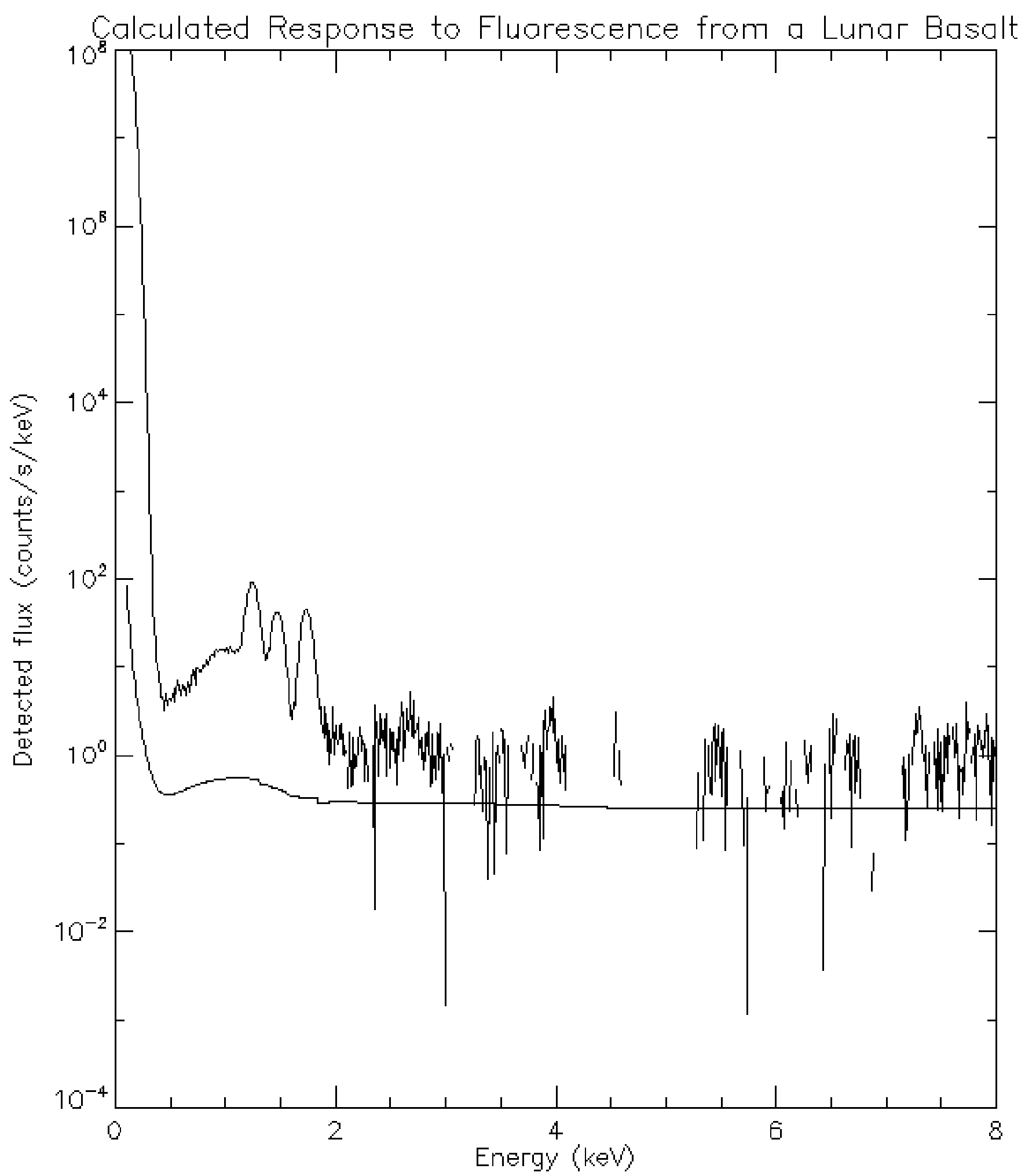

Figure 8 Calculated response to fluorescence from a representative Lunar basalt, using our physical instrument model, indicating the minimum detectable flux for $\mathrm{C} 1$ flare with a $14 \mathrm{deg}$ opening angle for a real detector area of $24 \mathrm{~cm} 2$ detector and a 0.8 throughput collimator expressed as counts/sec/keV and $100 \mathrm{eV}$ resolution. The calculation includes calibration and electronic efficiency data from D-CIXS The smooth line is the 3 sigma detection limit for a 16 second integration, typical for overflight of a single pixel. It is seen that the $\mathrm{Mg}, \mathrm{Al}$ and $\mathrm{Si}$ lines are well resolved in this baseline illumination condition. 


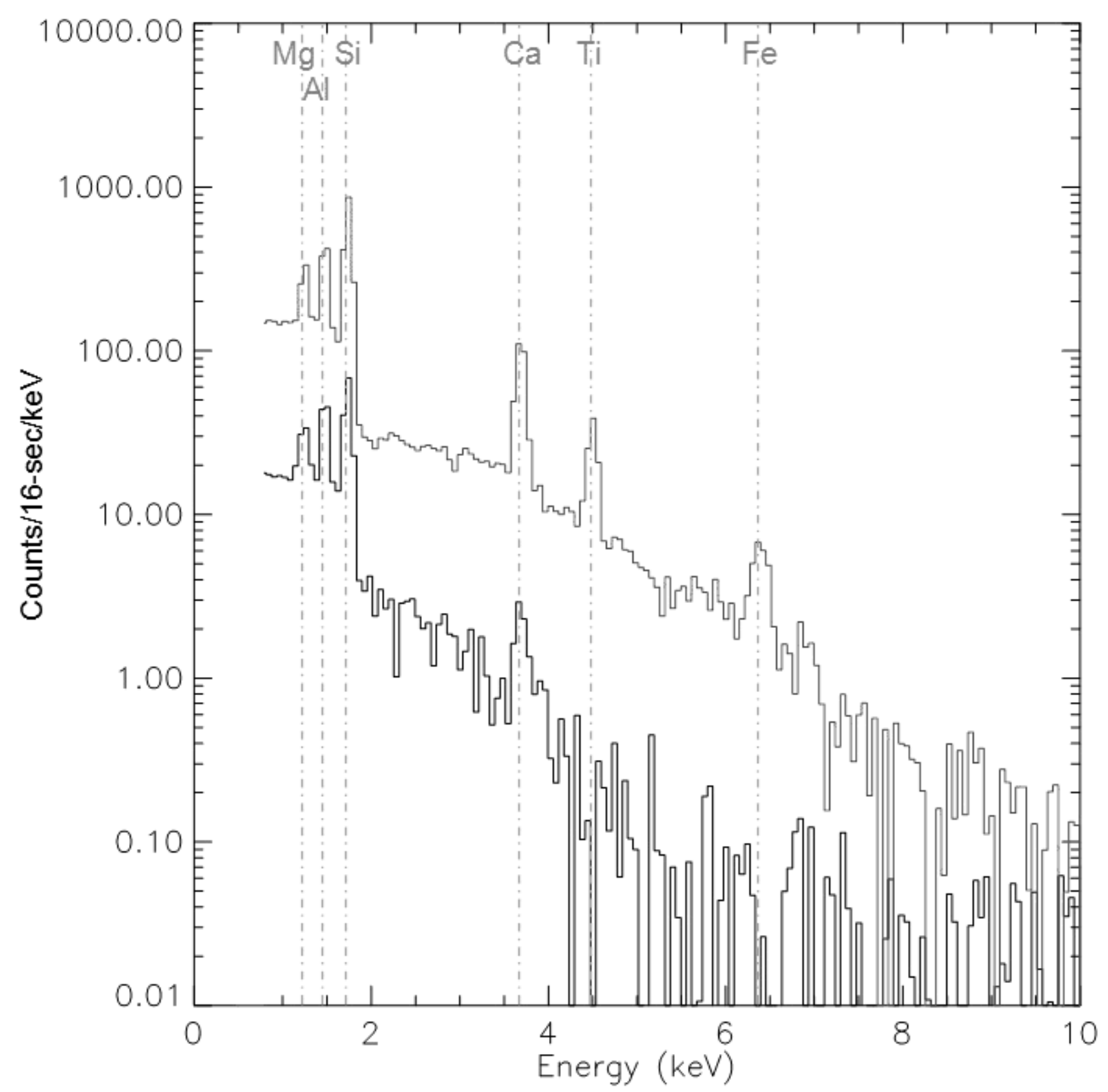

Figure 9. Simulated C1XS spectrum for the November 18 flare based on individual 16 second integrations. The lower line (black) shows the spectrum detected during the quiet period just before flare begins, while the upper line (grey) shows the spectrum obtained at the peak of the flare. 


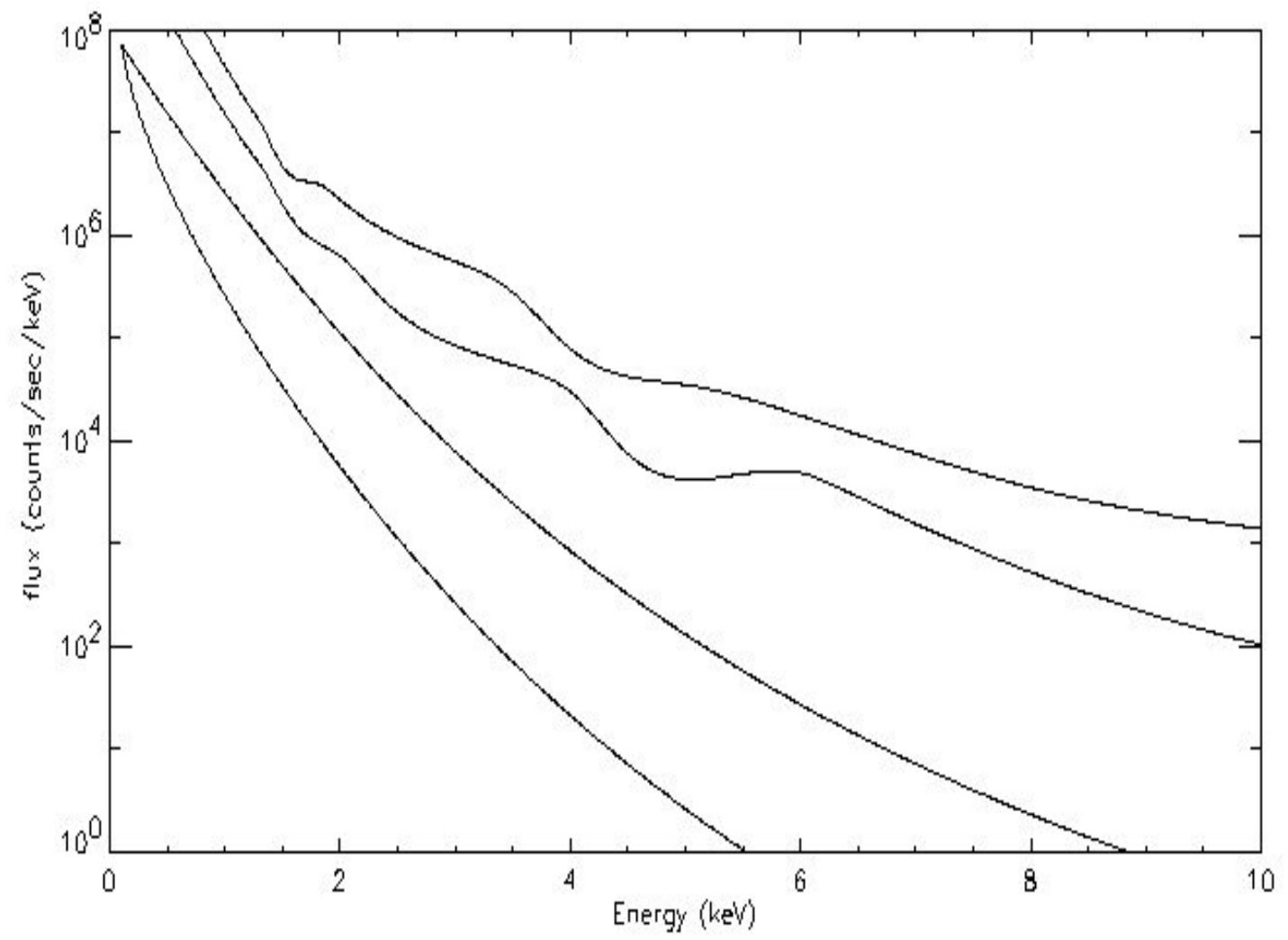

a)

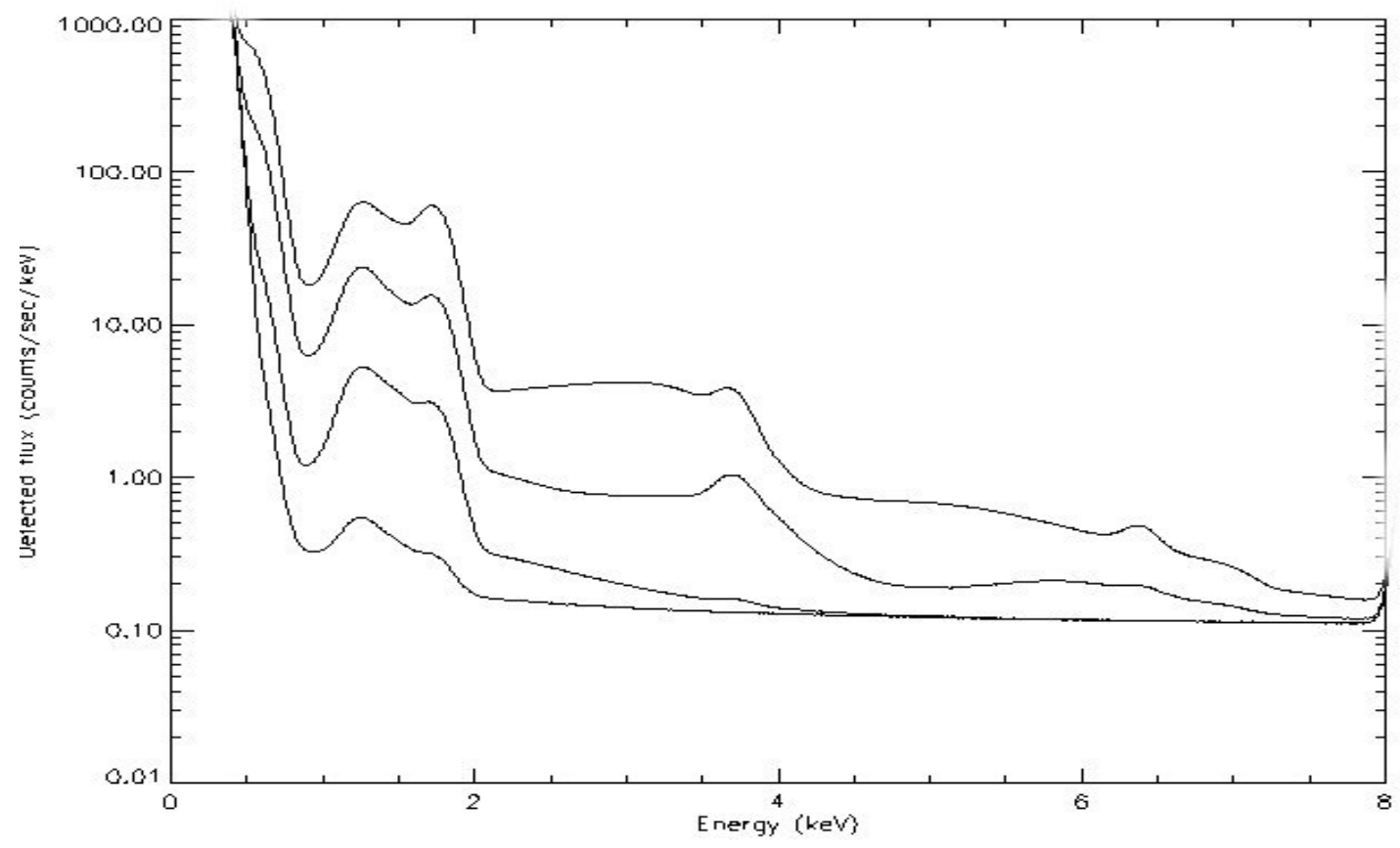

b)

Figure 10 Four different solar flare input levels (a5, b1,c1, m1), with the calculated lunar fluorescent spectra, as detected by C1XS, which would result. 


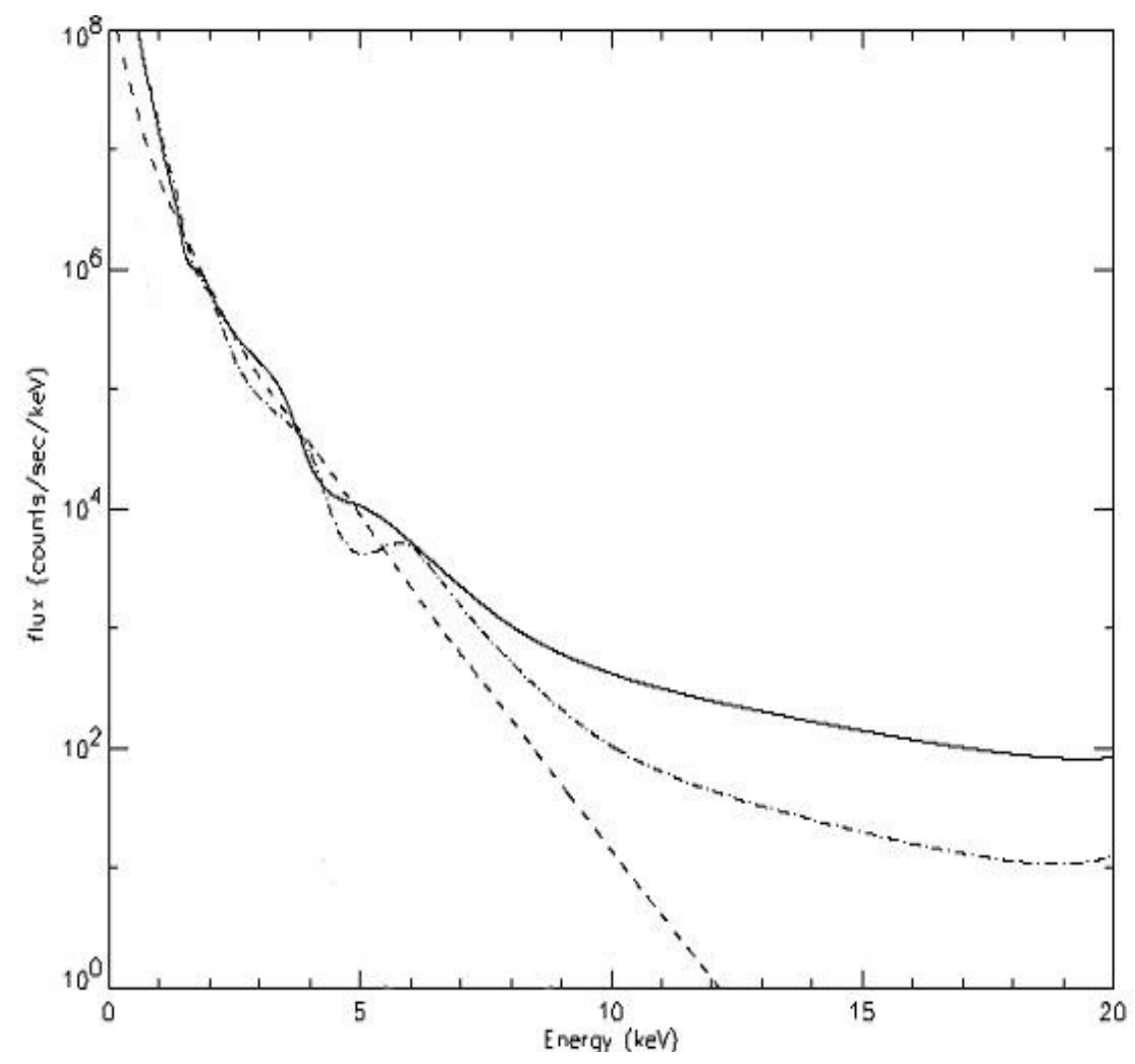

a)

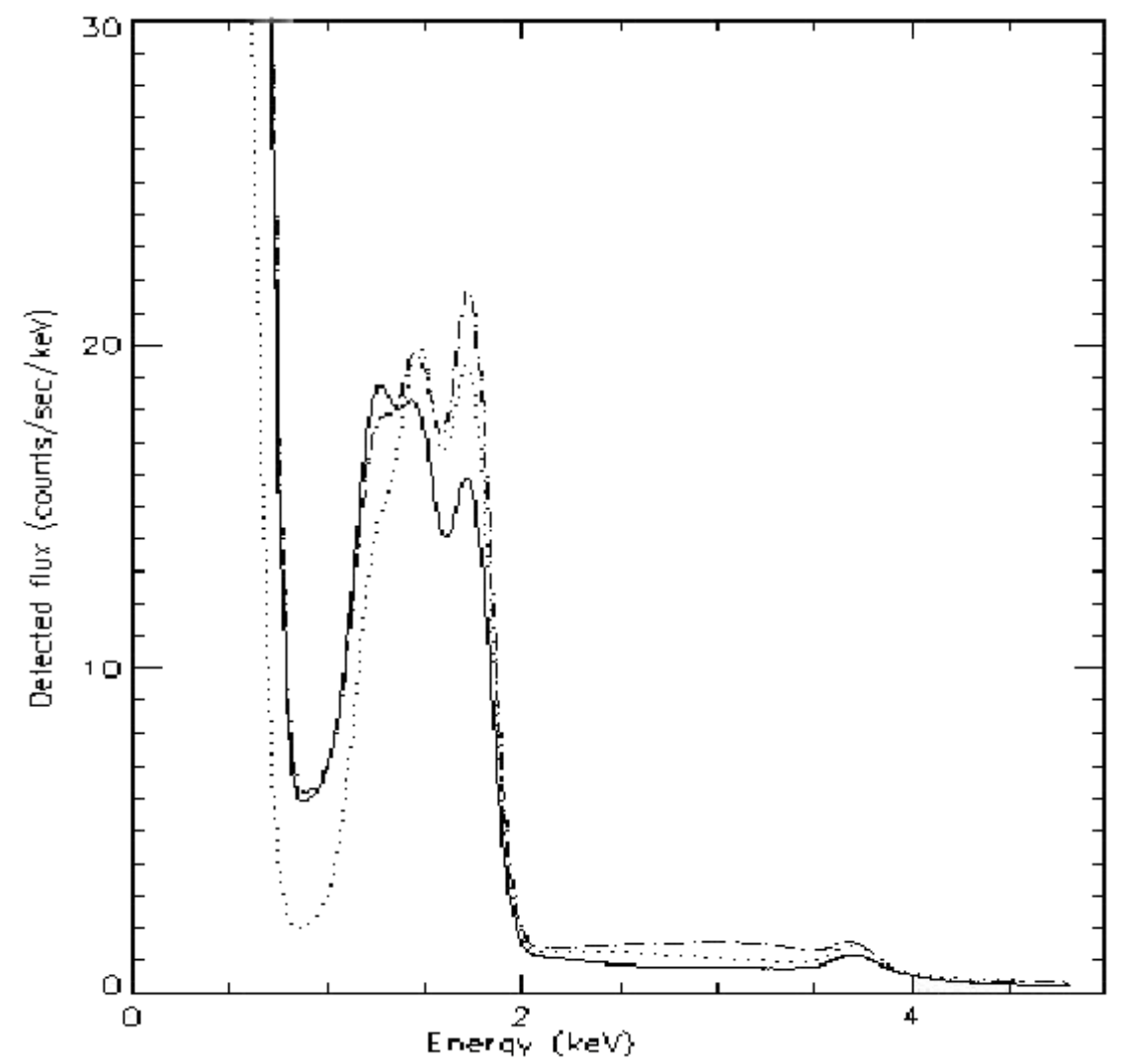

b)

Figure 11 Three different models of C1 solar flares (Mewe et al 1985, Clark et al 1997,) with the calculated lunar fluorescent spectra, as detected by C1XS, that would result. Note complete reversal of the line ratios which would be observed. 


\begin{tabular}{|l|l|l|}
\hline Element & $\mathbf{K} \alpha_{1}$ & $\mathbf{L} \alpha_{1}$ \\
\hline Oxygen & 524.9 & - \\
\hline Sodium & $1,040.98$ & - \\
\hline Magnesium & $1,253.60$ & - \\
\hline Aluminium & $1,486.70$ & - \\
\hline Silicon & $1,739.98$ & - \\
\hline Potasium & $3,313.8$ & - \\
\hline Calcium & $3,691.68$ & - \\
\hline Titanium & $4,510.84$ & - \\
\hline Iron & $6,403.84$ & 705.0 \\
\hline
\end{tabular}

Table 1 Energies $(\mathrm{KeV})$ of relevant X-ray fluorescent lines. See discussion for those lines detectable by C1XS. 


\section{THE C1XS X-RAY SPECTROMETER ON CHANDRAYAAN-1.}

M. Grande ${ }^{1}$, B. J. Maddison ${ }^{2}$, C.J. Howe ${ }^{2}$, B. J. Kellett ${ }^{2}$, P. Sreekumar ${ }^{3}$, J. Huovelin ${ }^{4}$, I. A. Craw-

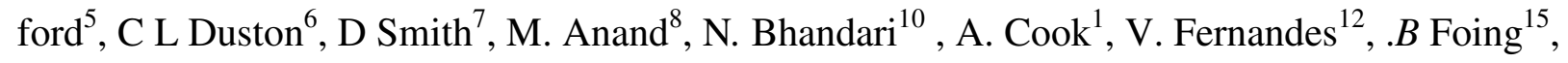
O Gasnaut $^{6}$, JN Goswami ${ }^{10}$, A Holland ${ }^{8}$, K. H. Joy ${ }^{2,5,9}$, D Kochney ${ }^{15}$, D Lawrence ${ }^{11}$, S Maurice ${ }^{6}$, T. Okada ${ }^{14}$, S Narendranath $^{3}$, C Pieters $^{16}$, D Rothery ${ }^{8}$, S. S. Russell ${ }^{9}$, A Shrivastava ${ }^{3}$, M Wiec- $^{-}$

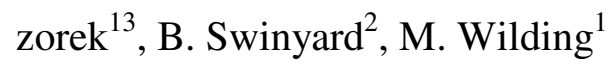

${ }^{1}$ Institute of Mathematical and Physical Sciences, University of Wales, Aberystwyth, SY23 3BZ, UK, M.Grande@aber.ac.uk, ${ }^{2}$ Rutherford Appleton Laboratory, Chilton, UK, ${ }^{3}$ Space Astronomy \& Instrumentation Division, ISRO Satellite Centre, Bangalore, India, ${ }^{4}$ The Observatory, Univ. of Helsinki, Finland, ${ }^{5}$ SThe Joint UCL/Birkbeck Research School of Earth Sciences, Gower Street, London, WC1E 6BT UK, ${ }^{6}$ Centre d'Etude Spatiale des Rayonnements, Université de Toulouse, CNRS, France, ${ }^{7}$ Brunel Univ, UK, ${ }^{8}$ Open University UK, ${ }^{9}$ Department of Mineralogy, Natural History Museum, Cromwell Road, London SW7 5BD, UK. UK. ${ }^{10}$ PRL India, ${ }^{11}$ Los Alamos National Lab, USA, ${ }^{12}$ Berkeley Geochronology Center, Berkeley, CA, USA, ${ }^{13}$ IPG Paris, France. ${ }^{14}$ ISAS/JAXA, Japan, ${ }^{15}$ ESTEC, ESA, Holland ${ }^{16}$ Brown University USA.

\section{Abstract:}

The Chandrayaan-1 X-ray Spectrometer (C1XS) is a compact X-ray spectrometer for the Indian Space Research Organisation (ISRO) Chandrayaan-1 lunar mission. It exploits heritage from the D-CIXS instrument on ESA's SMART-1 mission. As a result of detailed developments to all aspects of the design, its performance as measured in the laboratory greatly surpasses that of D-CIXS. In comparison with SMART-1, Chandrayaan-1 is a science oriented 
rather than a technology mission, leading to far more favourable conditions for science measurements. C1XS is designed to measure absolute and relative abundances of major rock-forming elements (principally $\mathrm{Mg}, \mathrm{Al}, \mathrm{Si}, \mathrm{Ca}$ and $\mathrm{Fe}$ ) in the lunar crust with spatial resolution $\leq 25$ FWHM km, and to achieve relative elemental abundances of better than $10 \%$.

\section{Introduction:}

The Chandrayaan-1 X-ray Spectrometer (C1XS) is a compact X-ray spectrometer for the Indian Space Research Organisation (ISRO) Chandrayaan-1 lunar mission, which was successfully launched on 22 October 2008. It exploits heritage from the D-CIXS instrument (Grande et al., 2001, 2003, 2007; Swinyard et al., forthcoming) on ESA's SMART-1 mission (Racca et al 2002). However, by comparison with SMART-1, Chandrayaan-1 is a science oriented rather than a technology mission, leading to far more favourable conditions for science measurements. C1XS is designed to measure absolute and relative abundances of major rockforming elements (principally $\mathrm{Mg}, \mathrm{Al}, \mathrm{Si}, \mathrm{Ca}, \mathrm{Ti}$ and $\mathrm{Fe}$ ) in the lunar crust with spatial resolution $\sim 25 \mathrm{~km}$.

The C1XS hardware was designed and built by an international team led from the Rutherford Appleton Laboratory (RAL), STFC. The Principal Investigator is Prof. M. Grande at Aberystwyth University. There is also a major science and design contribution from ISRO Satellite Centre, Bangalore, India; CESR, Toulouse, France provides 3-D Plus video processor integrated circuits, and there is an important contribution to the detector characterisation from Brunel University. The Science team is chaired by Dr. I. A. Crawford of Birkbeck College London. In order to record the incident solar X-ray flux at the Moon, C1XS carries an X-ray Solar Monitor 
(XSM) provided by the University of Helsinki Observatory, Finland. C1XS is primarily funded by ESA with partial support to RAL from ISRO.

D-CIXS was able to demonstrate an ability to sense remotely elements in the top few micrometers of the lunar regolith, in particular $\mathrm{Mg}, \mathrm{Al}, \mathrm{Si}, \mathrm{Ca}$ and $\mathrm{Fe}$ (Grande et al., 2007; Swinyard et al., forthcoming). The Ca detections represented the first unambiguous remote sensing of calcium. More recent detailed analysis shows that in favorable conditions titanium is also observed (Swinyard et al. forthcoming). Other companion papers describe in more detail the science goals (Crawford et al., forthcoming) the instrument construction (Howe et al., forthcoming) and the calibration status (Kellett et al., forthcoming).

\section{Instrument requirements}

Solar irradiation excites fluorescent emission from the lunar surface; by measuring this emission, whilst at the same time monitoring the incident solar X-ray emission, we are able to map the absolute elemental abundances of the main rock forming elements on the Moon. In addition, during bright flares, we detect localised concentration levels of key minor elements. The timing of the Chandrayaan-1 mission, ensuring that the spacecraft arrives at the beginning of the rising phase of the solar activity cycle, with near Solar maximum flux levels expected at the end of its nominal mission, is well suited for this purpose. The 10× higher solar X-ray fluxes, combined with the excellent ( $85-115 \mathrm{~km}$ near circular) orbit, will help ensure that C1XS can carry out enormously enhanced science compared to SMART-1.

The nominal mission duration is 2 years. Given the Moon's 28 day rotation, this corresponds to 25 daylight overflights for each $25 \mathrm{~km}$ FWHM Field of View on the surface, and 
16 within $60^{\circ}$ of zenith illumination. Illumination conditions will be different for each overflight, both for geometrical reasons, but much more importantly because of the huge variations in the solar X-ray illumination that take place on timescale of minutes, as shown in Figure 1b. At solar maximum, expected at or shortly after the end of the Chandrayaan-1 mission, X-ray illumination is above $\mathrm{C} 1$ category flare conditions for $\sim 40 \%$ of the time (see figure 2 ), based upon statistics from the previous cycle.. In a 2 year solar maximum mission, each pixel would be sampled with near zenith $\mathrm{C} 1$ illumination on average 6 times. A more precise calculation shows around 95\% probability of a pixel being illuminated at greater than $\mathrm{C} 1$ at some point during the mission, which is sufficient to return the required spectral resolution. Around $10 \%$ of pixels should be illuminated with greater than M1 at some point during the mission. We note that C1XS is to be launched at around the beginning of solar cycle, and that fluxes are therefore very sensitive to variations of a few months in the upturn in the solar cycle relative to the launch date. Figure 2 is based on the current (27 Jun 2008) best NOAA SEC predictions (Biesecker 2008) showing the high and low predictions. Currently (Keating, 2008) the cycle appears to be an average 11 year cycle. A 6 month mission extension, at full Solar maximum, would certainly yield large increases in the quality of $\mathrm{x}$-ray illumination.

We can investigate minor elements like sodium, phosphorous and sulphur which provide great insight into lunar evolution. The energy range of CIXS is 0.8 to $7 \mathrm{keV}$, and the energy resolution at launch is $\sim 160 \mathrm{eV} \mathrm{FWHM}$ at $8 \mathrm{KeV}(2 \%)$, sufficient to resolve all the main fluorescence lines of interest, as shown in table 1. The ability to detect sodium ( $\mathrm{K} \alpha$ at 1.043 $\mathrm{keV}$ ) if it is present in significant quantities is particularly interesting. It may also be possible to detect the iron L-lines, which will enable C1XS to observe iron in all illumination conditions. For these reasons, particular care has been taken in defining the lower energy cut-off, as illus- 
trated in figure 3a. The low energy discriminator level is software commandable, but whilst in theory it could be lowered to include the oxygen $\mathrm{K} \alpha$ line at $525 \mathrm{keV}$, and the detectors have some sensitivity at these low energies, the filter cutoff shown in figure $3 \mathrm{~b}$ would preclude useful information. Since the oxygen concentration across the highly oxidised lunar surface does not vary outside a range $41 \%$ - 46\% (eg Lawrence et al), this data would not in any case yield significant new information. However, at start of mission, we will have sufficient sensitivity for the Fe L $\alpha$ line at $705 \mathrm{eV}$ which greatly improves the functionality of the instrument, enabling Fe concentrations to be measured in all illumination conditions. Figure $3 \mathrm{c}$ shows the calculated overall effective area of the instrument, excluding electronic considerations.

In order to obtain good absolute elemental abundances by the X-ray fluorescence technique, it is essential to continuously monitor the solar X-ray flux which excites the lunar emission. To this end the CIXS instrument includes an X-ray Solar Monitor (XSM), designed and delivered by the University of Finland. The XSM will also provide a scientific bonus in providing a long time series of the solar X-ray spectra with high spectral resolution and full energy band coverage.

\section{Instrument}

The baseline instrument design (see figure 4) consists of 24 nadir pointing Swept Charge Device (SCD) detectors (Howe et al., forthcoming). A traditional box collimator defines the field of view of each SCD, resulting in a triangular angular sensitivity with $50 \%$ of the X-ray signal deriving from $14^{\circ}$ of the collimator aperture, corresponding to $25 \mathrm{~km}$ on the lunar surface from Chandrayaan-1's circular $100 \mathrm{~km}$ orbit. Due to the highly elliptical orbit of SMART-1 the corresponding values for D-CIXS ranged from 32 to $315 \mathrm{~km}$. The uniform spatial resolution of 
C1XS will greatly simplify the data analysis. The C1XS collimator stack differs from that on DCIXS in that it is machined numerically, as opposed to by lithographic construction (Grande et al 2003, Howe et al forthcoming). Figure 5 shows the flight instrument during calibration.

A deployable door protects the instrument during launch and cruise, and also provides a ${ }^{55} \mathrm{Fe}$ calibration $\mathrm{X}$-ray source for each of the detectors, allowing in flight calibration to be performed. The source strength is sufficient over the two year mission for gain calibration to the required $1 \%$ accuracy to be obtained within 10 minutes. This will also allow energy and FWHM calibrations of sufficient accuracy to be obtained.

\section{Detectors}

The Swept Charge Device (SCD) detectors (Gow et al 2007) provide high detection efficiency in the 0.8 to $7 \mathrm{keV}$ range, which contains the $\mathrm{X}$-ray fluorescence lines of interest. The SCD is a CCD-like device which achieves near Fano-limited spectroscopy below -10 C. It has a continuous one dimensional readout architecture which is otherwise similar to a conventional $\mathrm{CCD}$, and a $1.1 \mathrm{~cm} 2$ detector area. The instrument design aims to keep detector temperatures below $-17.5^{\circ} \mathrm{C}$, which provides sufficiently low SCD leakage current to ensure optimum signal to noise and stability, as well as improving radiation tolerance.

The detectors are shielded from the lunar UV and visible albedo, as well as protons below $180 \mathrm{keV}$ and low energy electrons, by two layers of $400 \mathrm{~nm}$ aluminized polyimide filtering (figure $3 \mathrm{~b}$ shows their calculated $\mathrm{x}$-ray transmission). Careful thought has been given to the radiation shielding, in what is already a comparatively low radiation environment orbit. It will now consist of a $4 \mathrm{~mm}$ thick aluminum electronics box with $3 \mathrm{~mm}$ of copper and $6 \mathrm{~mm}$ of tantalum behind the SCD modules. Due to the low altitude, the spacecraft is well shielded from the 
139 front by the Moon itself. The collimator structure and additional tantalum provides additional 140 shielding for oblique angles.

141

The principal instrument requirement is a spectral resolution sufficient to clearly resolve the three common light rock forming elements $(\mathrm{Mg}, \mathrm{Al}, \mathrm{Si})$ As will be seen from table 1 below, this implies an energy resolution better than $250 \mathrm{eV}$ at 1-2 keV. Figures $6 \mathrm{a}$ and $6 \mathrm{~b}$ indicates that in laboratory calibration this condition is comfortably met. The effects of radiation tests on SCD detectors from the same batch are shown in Figure 7 suggesting that even at end of life, the performance requirements will be met. There is some uncertainty in the predicted range of exposures due to the sensitivity to the phase of the solar cycle. The figures shown reflect the fact that as of the present, large solar flares have not been observed in the current rising cycle. Note by comparison the reduced energy resolution of D-CIXS after the heavy radiation doses it incurred during its extended cruise phase to the Moon (Grande et al., 2007).

The maximum expected count rate for the C1XS instrument will be 2000 counts per second for all 24 detectors, for an X20 flare, acceptably within the instrument limit of 5500 cps (see Howe et al., forthcoming). Additional refinements to the electronics, onboard software and thermal design will also greatly increase detector stability and signal to noise ratio over what was achieved on D-CIXS [Grande et al., 2007]. Electronic noise has been reduced to 60eV. A detailed account of the technical development is given in Howe et al.,(forthcoming).

\section{X-ray Solar Monitor}

The X-ray solar monitor (XSM) is based on the SMART-1 XSM (Huovelin et al 2002) and consists of a separate silicon detector unit on the spacecraft. The non-imaging HPSi PIN sensor has a wide field-of-view (FOV) to enable Sun visibility during a significant fraction 
of the mission lifetime, which is essential for obtaining calibration spectra for the X-ray fluorescence measurements by the C1XS spectrometer. The energy range (1-20 keV), spectral resolution (about $200 \mathrm{eV}$ at $6 \mathrm{keV}$ ), and sensitivity (about $7000 \mathrm{cps}$ at flux level of $10^{-4} \mathrm{~W} \mathrm{~m}^{-2}$ in the range $1-8 \mathrm{keV}$ ) are tuned to provide optimal knowledge about the solar X-ray flux, matching well with the activating energy range for the fluorescence measured by C1XS.

As has been remarked, the $\mathrm{X}$-ray flux rises rapidly during a major solar flare. However, this is frequently followed by an increase in penetrating background radiation, at a time delay dependent on the energy and the geometry of the interplanetary magnetic flux. Thus it is still in general possible to use the brightest X-class events for fluorescence spectroscopy, and the very high fluorescence count rates obtained will be invaluable in revealing the concentrations of minor elements in the regolith. Typical time delays are of up to one hour duration.

\section{Predicted Response:}

The baseline specification is to achieve $10 \%$ relative elemental abundance accuracy from a single overflight of a $25 \mathrm{~km}$ pixel in $\mathrm{C} 1$ solar flare conditions, and we consider the instrument response in terms of this baseline situation. Figure 8 shows the calculated response to fluorescence from a representative Lunar basalt, using our physical instrument model, indicating the minimum detectable flux for $\mathrm{C} 1$ flare with a 14 deg opening angle for a real detector area of $24 \mathrm{~cm}^{2}$ detector and a 0.8 throughput collimator expressed as counts $/ \mathrm{sec} / \mathrm{keV}$ and a $100 \mathrm{eV}$ resolution. The calculation includes calibration and electronic efficiency data from D-CIXS. The smooth line is the 3 sigma detection limit for a 16 second integration, typical for overflight of a single pixel. It is seen that the $\mathrm{Mg}, \mathrm{Al}$ and $\mathrm{Si}$ lines are well resolved for this baseline illumination condition. As an example of inferred performance under flare conditions, Figure 9 shows a com- 
parison of predicted C1XS response in quiet and flare conditions to an actual event observed by D-CIXS, described in the accompanying paper by Swinyard et al., (forthcoming). Note the greatly increased signal for the low-energy $\mathrm{Mg}, \mathrm{Al}$ and $\mathrm{Si}$ lines, and the excellent signal-to-noise ratio in the $\mathrm{Ca}, \mathrm{Ti}$ and $\mathrm{Fe}$ lines at the peak of the flare. Again, instrument response $(100 \mathrm{eV})$ is derived from C1XS laboratory calibrations (Kellett et al., forthcoming).

Accurate knowledge of the input solar spectrum is essential for determination of elemental abundances. A linear difference in solar input will leave the relative line ratios unchanged. However, this is not the case if the shape of the input solar spectrum changes. Figure 10 shows 4 different flare levels (a5, b1, c1, m1), with the output spectra that would result. We note that the apparent line ratios are very significantly modified. The point is made even more clearly in Figure 11 which shows three different models of C1 solar flares (Mewe et al., 1985, Clark et al., 1997,). The calculated lunar fluorescent spectra, which would be detected following scintillation of lunar basalt, are also shown. In this case the predicted line ratios are modified by more than $+/-10 \%$.

This emphasizes the vital importance of accurate monitoring of the solar input spectrum, as well as good codes to forward model the expected lunar X-ray fluorescence for different possible regolith compositions. Thus, whilst elemental abundance ratios may be useful diagnostics in our initial analysis, final estimations of lunar elemental abundance ratios will require detailed modelling (see Swinyard et al., forthcoming). One of the lessons learned from D-CIXS was the critical importance of fully characterizing the input solar spectrum, if one is to derive absolute lunar elemental surface abundances. In comparison to D-CIXS, C1XS and XSM are far better calibrated. Details of the results obtained in the calibration campaign of the C1XS instrument are given by Kellett et al. (forthcoming). 


\section{Science Goals:}

A detailed description of the science objectives for the instrument and the match of its capabilities to key questions is given in a companion paper by Crawford et al., (in press). C1XS will arrive at the Moon in the run up to the maximum of the solar cycle, and the high incident X-ray flux observed from an orbit optimized for science, and coupled with good instrumental energy resolution, means that we will obtain composition data accurate to better than $10 \%$ of major elemental abundances over the entire surface. We note that observations of major element abundances for regions where samples have been obtained by the Apollo and Luna missions will

be used to validate the calibration of C1XS measurements. Thus, C1XS will be well-placed to make significant contributions to lunar science in a number of areas.

Specifically, C1XS will determine the major element geochemistry (and especially $\mathrm{Mg} / \mathrm{Si}$ and/or $\mathrm{Mg} / \mathrm{Fe}$ elemental ratios) in the main lunar terrain types (i.e. Procellarum KREEP Terrane, South Pole-Aitken Basin, and the Farside Highlands; Jolliff et al., 2000) and establish the geographical distribution of the magnesian suite of rocks. A key ambition is to determine the large-scale stratigraphy of lower crust (and possibly crust/mantle boundary region) by measuring the elemental abundances of the floor material of large basins not obscured by mare basalts (e.g. SPA and other farside basins), and the central rings and ejecta of large basins which expose material derived from depths of many tens of $\mathrm{km}$. In addition, determination of the crustal aluminium abundance and distribution is important for the assessment of lunar refractory element enrichment, and C1XS-derived aluminium abundance maps will thus constrain models of lunar origins. Last but not least, the $\sim 25 \mathrm{~km}$ spatial resolution will enable C1XS to address a number of smaller-scale geological issues (e.g., the composition of discrete mare basalt lava flows and, py- 
231 roclastic deposits) which also refine our understanding of lunar geological evolution (Joy et al., 232 2008, Crawford et al., in press).

\section{Conclusions} work provided by the ISRO Chandrayaan-1 mission to the Moon. This is highly suitable for producing high quality data on lunar composition derived from Lunar X-ray fluorescence spectra, taken in the approach to Solar maximum. The instrument represents a considerable refinement on

240 the original D-CIXS instrument on SMART-1. It is expected to provide data of the spatial and 241 spectral resolution required to produce significant progress in lunar science. 


\section{Acknowledgements}

The C1XS instrument development was supported with funding from ESA Science and Technology Research Programmes. Major thanks for support are due to RAL/STFC, and also ISRO ISAC. Additional hardware was provided by CESR, Toulouse, and University of Helsinki Observatory. J Carter of Aberystwyth University for recalculating figure 8.

\section{References:}

Biesecker D, and the NOAA/SEC Solar Cycle 24 Panel . The Solar Cycle 24 Consensus Prediction; Web document www.swpc.noaa.gov/SolarCycle/SC24/Biesecker.ppt June 2008

Clark P E and J.I. Trombka J. Geophys. Res. 102, 16631 (1997)

Crawford I A , K.H. Joy, B.J. Kellett, M. Grande, M. Anand, N. Bhandari, A.C. Cook, L. d’Uston, V.A. Fernandes, O. Gasnault, J. Goswami, C.J. Howe, J. Huovelin, D. Koschny, D.J. Lawrence, B.J. Maddison, S. Maurice, S. Narendranath, C. Pieters, T. Okada, D.A. Rothery, S.S. Russell, P. Sreekumar, B. Swinyard, M. Wieczorek, M. Wilding' The Scientific Rationale for the C1XS X-Ray Spectrometer on India's Chandrayaan-1 Mission to the Moon, Planet. Space. Sci., (submitted PSS 2008 This volume 2008).

Gow, J; Smith, DR; Holland, AD; Maddison, B; Howe, C; Sreekumar, P; Huovelin, J; Grande, M, Characterisation of swept-charge devices for the Chandrayaan-1 X-ray Spectrometer (C1XS) 
264

265

266

267

268

269

270

271

272

273

274

275

276

277

278

279

280

281

282

283

284 285 instrument - art. no. 66860I, OHW, UV, X-RAY, AND GAMMA-RAY SPACE

INSTRUMENTATION FOR ASTRONOMY XV 6686I6860-I6860 2007

Grande M The D-CIXS X-ray Spectrometer on ESA's SMART-1 mission to the Moon EARTH MOON AND PLANETS 2001, Vol 85-6, pp 143-152

Grande M., et al, The D-CIXS X-ray mapping spectrometer on SMART-1, Planetary and Space Science, 2003, 51, 427-433,

Grande M., et al. The D-CIXS X-ray spectrometer on the SMART-1 mission to the Moon-First Results. Planetary and Space Science 55, 494 (2007)

Howe et al (submitted PSS 2008 This volume 2008).

Huovelin J; Alha L; Andersson H; Andersson T; Browning R; Drummond D; Foing B; Grande M; Hamalainen K; Laukkanen J; Lamsa V; Muinonen K; Murray M; Nenonen S; Salminen A; Sipila H; Taylor I; Vilhu O; Waltham N; Lopez-Jorkama M The SMART-1 X-ray solar monitor (XSM): calibrations for D-CIXS and independent coronal science. PLANETARY AND SPACE SCIENCE 2002, Vol 50, Iss 14-15, pp 1345-1353

Jolliff, B.L., Gillis, J.J., Haskin, L.A., Korotev, R.L., Wieczorek, M.A. 2000. Major lunar crustal terranes: surface expressions and crust-mantle origins. J. Geophys. Res. 105, 4197 
Joy K. H., Crawford I.A., Kellett B., Grande M.N. and the C1XS Science Team. (2008). The Scientific Case for the Chandrayaan-1 X-Ray Spectrometer. In Lunar and Planetary Science XXXVIX, abstract no. 1070, 39th Lunar and Planetary Science Conference, Houston.

Keating 2008 EOS in press

Kellett et al (submitted PSS 2008 This volume 2008).

Lawrence D. J., W. C. Feldman, B. L. Barraclough, A. B. Binder,

R. C. Elphic, S. Maurice, D. R. Thomsen, Global Elemental Maps of the

Moon: The Lunar Prospector Gamma-Ray Spectrometer 4 SEPTEMBER 1998 VOL 281

SCIENCE 1998

R. Mewe, E. H. B. M. Gronenschild, G. H. J. van den Oord, V. Astron. Astrophys. Supp. 62, 197. (1985)

Racca GD, Marini A, Stagnaro L, van Dooren J, di Napoli L, Foing BH, Lumb R, Volp J, Brinkmann J, Grunagel R, Estublier D, Tremolizzo E, McKay M, Camino O, Schoemaekers J, Hechler M, Khan M, Rathsman P, Andersson G, Anflo K, Berge S, Bodin P, Edfors A, Hussain A, Kugelberg J, Larsson N, Ljung B, Meijer L, Mortsell A, Nordeback T, Persson S, Sjoberg F. SMART-1 mission description and development status. PLANETARY AND SPACE SCIENCE Volume: 50 Issue: 14-15 Pages: 1323-1337 Published: DEC 2002 
309 Swinyard et at (submitted PSS 2008 This volume 2008).

310 